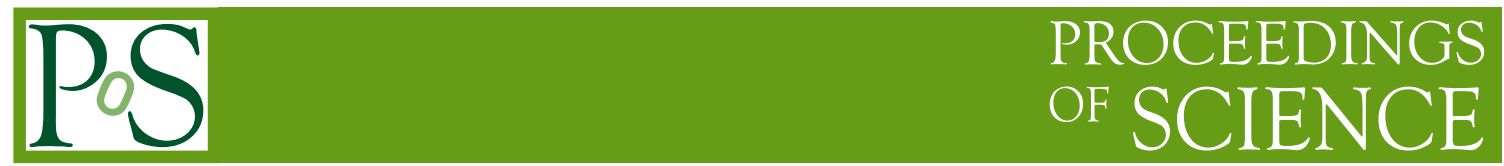

\title{
Searches for new physics in high-mass fermionic final states and jets with the ATLAS detector at the LHC
}

\section{Oleksandr Viazlo*}

On behalf of the ATLAS Collaboration.

Lund University (SE)

E-mail: oleksandr.viazlodcern.ch

\begin{abstract}
Many new physics scenarios beyond the Standard Model predict resonant or non-resonant production of high-transverse momentum fermions or gluons. This report summarizes Run 2 searches for new physics in fermionic final states and jets with the ATLAS experiment at the LHC.
\end{abstract}

XXIV International Workshop on Deep-Inelastic Scattering and Related Subjects

11-15 April, 2016

DESY Hamburg, Germany

\footnotetext{
* Speaker.
} 


\section{Introduction}

In 2015, the center of mass energy of the Large Hadron Collider (LHC) increase from $\sqrt{s}=$ $8 \mathrm{TeV}$ to $\sqrt{s}=13 \mathrm{TeV}$, allowing to explore a new energy regime. Many searches for new physics were performed, targeting high-mass final states. In this paper, searches for the Beyond the Standard Model (BSM) signatures in fermionic, hadronic and mixed fermionic-hadronic final states are presented. All searches were performed with data collected by the ATLAS detector in 2015 at $\sqrt{s}=13 \mathrm{TeV}$, corresponding to $3.0-3.6 \mathrm{fb}^{-1}$ of luminosity.

There are many models which address questions of dark matter, neutrino mass, naturalness and other BSM aspects. And these models introduce new states at the $\mathrm{TeV}$ scale, which can potentially be discovered at LHC.

\section{Leptonic final states}

This report covers searches for three leptonic final states: one lepton and non-observable particle(s) [1], di-lepton same-flavor [2] and di-lepton different-flavor (electron-muon pair) [3]. The signatures for these searches are high- $p_{T}$ isolated leptons, as well as a substantial missing transverse momentum $E_{T}^{\text {miss }}$ for one of the searches. The Standard Model (SM) background in these searches is described by the Monte Carlo (MC) simulations with higher order corrections: cross section of charged and neutral current Drell-Yan (DY) processes were corrected by next-to-next-to-leadingorder (NNLO) QCD and next-to-leading-order (NLO) electroweak (EW) corrections, single top background was corrected by NNLO QCD corrections, and all other background processes were corrected by NLO QCD corrections. Description of these models are detailed in Ref. [1, 2, 3]. The multijet background component is estimated by the data-driven "matrix" method [1].

The search variable for the lepton plus $E_{T}^{\text {miss }}$ final state is the transverse mass $m_{\mathrm{T}}$ of the lepton and $E_{T}^{\text {miss }}$ :

$$
m_{\mathrm{T}}=\sqrt{2 p_{\mathrm{T}} E_{\mathrm{T}}^{\text {miss }}\left(1-\cos \varphi_{\ell v}\right)}
$$

where $\varphi_{\ell v}$ is the angle between the lepton and $E_{T}^{m i s s}$ in the transverse plane. For the two other final states, the search variable is the invariant mass of the di-lepton system. The strategy of the analysis is to look for a deviation of the observed data from the predicted SM background in the spectrum of the search variable.

For the lepton plus $E_{T}^{\text {miss }}$ analysis, only one isolated electron (muon) is required, with $p_{T}>$ $65(55) \mathrm{GeV}$ and with an identical requirement on $E_{T}^{m i s s}$. For the di-lepton analyses, two isolated leptons are required with $p_{T}>30 \mathrm{GeV}$.

$W^{\prime}$ and $Z^{\prime}$ Sequential Standard Models (SSM) [4] are used as benchmark models for these searches. Within these models, $W^{\prime}$ and $Z^{\prime}$ are assumed to be heavier copies of the SM $W$ and $Z$ bosons, with the same couplings. Other BSM models, such as the extra dimensions, contact interaction and E6 Grand Unified Theory models, were tested [1, 2, 3], but are not covered in this report.

The transverse mass distribution $m_{\mathrm{T}}$ with resonant $W^{\prime}$ signals for several masses overlaid is shown in Figure 1 (left panel). An overall good agreement between data and the background 
expectation can be seen. In Figure 1 (right panel), upper limits on the cross-section times branching ratio are shown. Expected and observed $W^{\prime}$ mass limits are set, and correspond to 4.18 and $4.07 \mathrm{TeV}$, respectively.
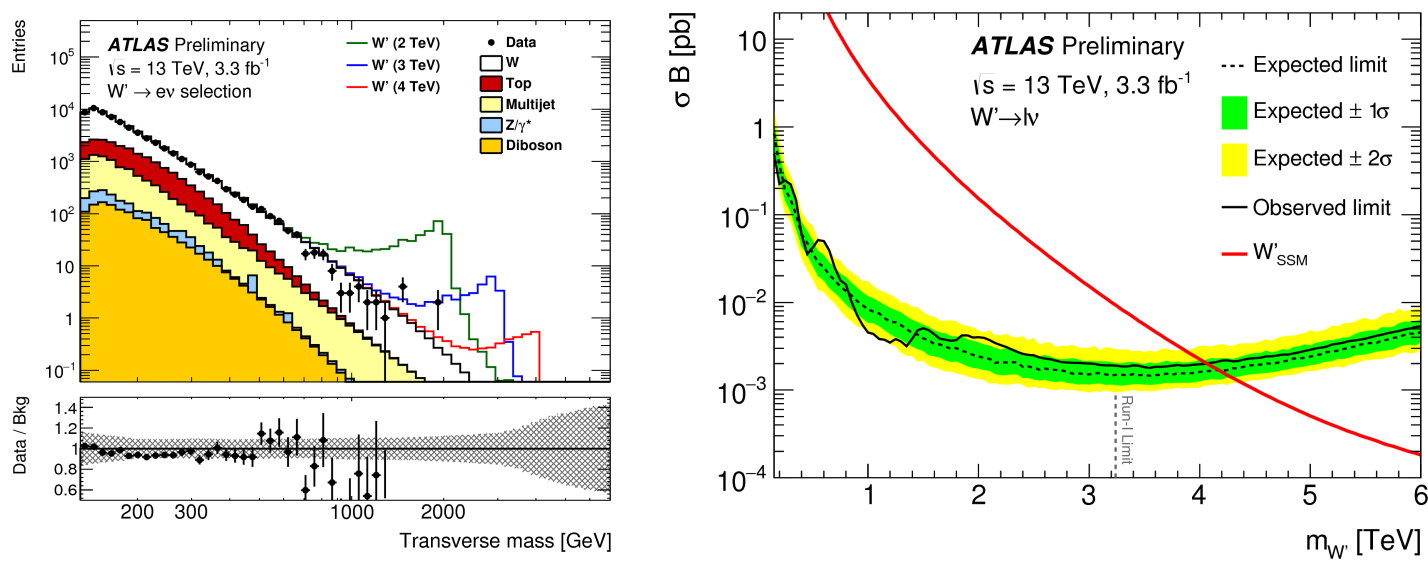

Figure 1: $m_{\mathrm{T}}$ spectrum of the electron channel (left panel) and the $W^{\prime}$ cross section limit for the combined electron and muon channels (right panel) of search in the lepton plus $E_{T}^{\text {miss }}$ channel [1].

In Figure 2, upper limits on the cross-section times branching ratio for the same-flavor (left panel) and the different-flavor (right panel) di-lepton final states are shown. Expected and observed $Z^{\prime}$ mass limits are set, and correspond to 3.37 (3.19) and 3.40 (3.01) TeV, respectively, for the sameflavor and the different-flavor di-lepton final states.
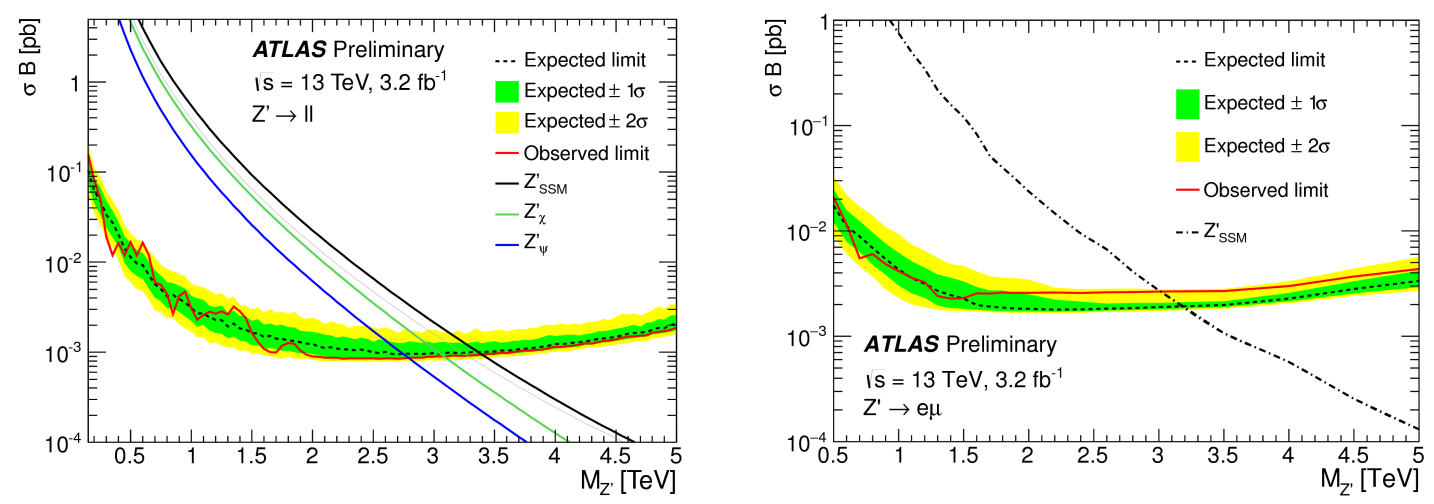

Figure 2: Upper limits on the $Z^{\prime}$ cross-section times branching ratio for the same-flavor (left panel) [2] and the different-flavor (right panel) [3] di-lepton final states.

\section{Hadronic final states}

Hadronic final state searches covered in this report are: di-jet [5], di-jet with b-tagging [6], and multijet [7] final states.

The di-jet analysis signature is at least two jets with $p_{\mathrm{T}}>440(50) \mathrm{GeV}$. The search variable of the analysis is the invariant mass $m_{j j}$ of the two leading jets. The analysis strategy is to look 
for localized excesses with respect to a background-only fit. Due to the fact that QCD predicts a smoothly falling $m_{j j}$ spectrum, the SM background is modelled with a power law function:

$$
f(z)=p_{1}(1-z)^{p_{2}} z^{p_{3}}
$$

where $z \equiv m_{j j} / \sqrt{s}$. Many BSM models predict excesses in the di-jet spectrum [8]. This study considered quantum black holes [9], excited quarks [10], $W^{\prime}$ and $Z^{\prime}$ bosons [4]. These models would produce peaks in the $m_{j j}$ distribution. The invariant mass $m_{j j}$ with resonant quantum black hole and excited quark signals overlaid is shown in Figure 3 (left panel). In Figure 3 (right panel), upper limits on the cross-section for all considered BSM models are shown. As a complement to this analysis, a di-jet angular analysis was done [5], but is not covered in this report.
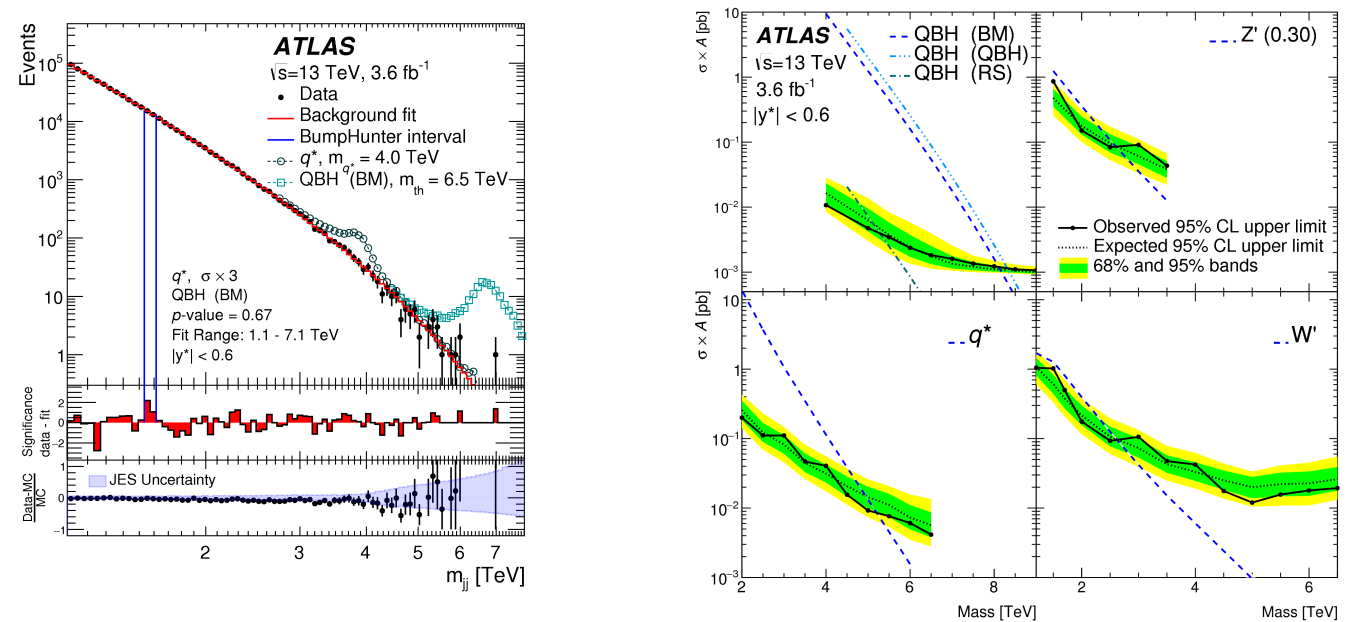

Figure 3: The reconstructed di-jet mass distribution (left panel). The solid line depicts the fit with Eq. 3.1, as discussed in the text. Predictions for an excited quark and a quantum black hole signals are shown above the fit. The lower panel shows the relative differences between the data and the prediction of PYTHIA 8 simulation of QCD processes, corrected for NLO and electroweak effects, and is shown purely for comparison. The $95 \%$ credibility-level upper limits are shown for quantum black holes, $Z^{\prime}$, excited quark and $W^{\prime}$ (right panel) [5].

The di-jet analysis with b-tagging exploits the same idea and selection criteria as the di-jet analysis, but with additional requirements of at least one or strictly two jets to be b-tagged. The invariant di-jet mass spectrum with at least one jet being b-tagged is shown in Figure 4 (left panel). No evidence of a significant excess of events is found compared to the expectations of the SM. Main BSM models of interest in this study are the excited b-quark [10] and the leptophobic $Z^{\prime}$ [11] ones. Upper limits on the cross-section times branching ratio for the excited b-quark model are shown in Figure 4 (right panel). Excluded mass regions for the excited b-quark (leptophobic $Z^{\prime}$ ) correspond to 1.1-2.1 (1.1-1.5) TeV, respectively.

The last hadronic final state analysis presented in this report is the multijet state. The search variable is the scalar sum of the jet $p_{T}\left(H_{T}\right)$. The analysis signature constitutes at least three jets with $p_{T}>50 \mathrm{GeV}$ and $H_{T}>1 \mathrm{TeV}$. The background model relies on a fit-based technique to extrapolate from low- $H_{T}$ control region (CR) to a high- $H_{T}$ signal region (SR) to estimate the 

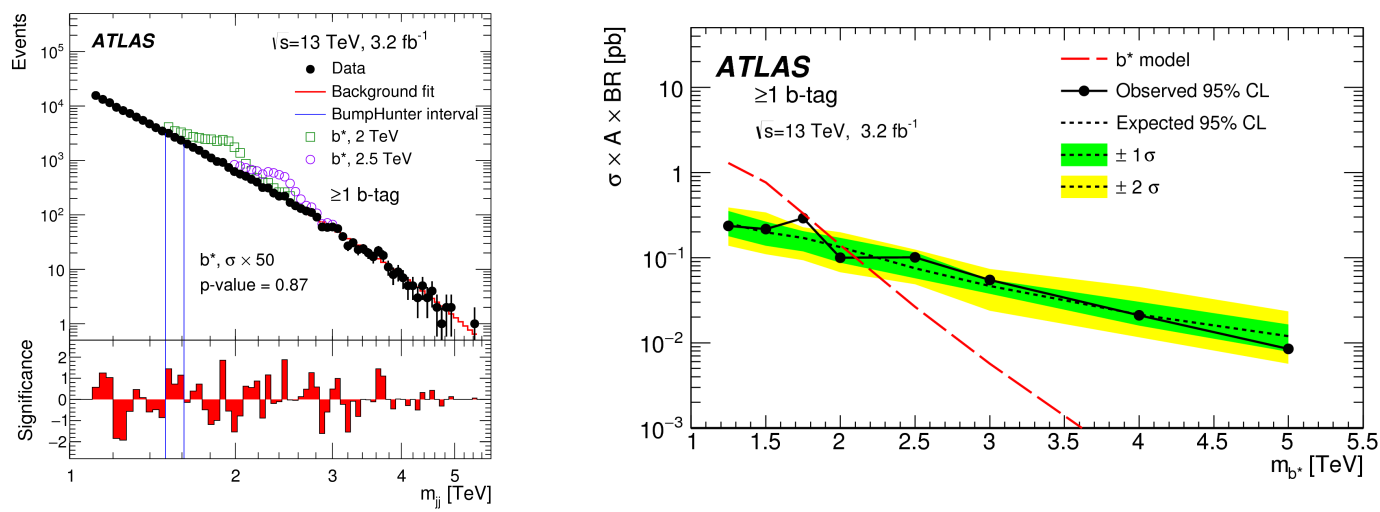

Figure 4: The reconstructed di-jet mass distribution with at least one jet b-tagged (left panel). The solid line depicts the background prediction, as discussed in the text. Predictions for an excited b-quark signal are shown above the fit. The $95 \%$ credibility-level upper limits are shown for the excited b-quark (right panel) [6].

amount of the SM background where contributions of new physics are expected. A medium- $H_{T}$ validation region (VR) is used to validate the fit. The three regions are shown in Figure 5 (left panel). BSM signal models considered in the analysis were the rotating black holes (with two, four and six extra dimensions) and the string balls models. Upper limits on the cross-section times branching ratio for the rotating black holes model are shown in Figure 5 (right panel). The production of a rotating black hole with six extra dimensions is excluded for a mass threshold $\left(M_{t h}\right)$, from which black holes start to form, up to 9.0-9.7 TeV, depending on the fundamental Planck scale $\left(M_{D}\right)$.
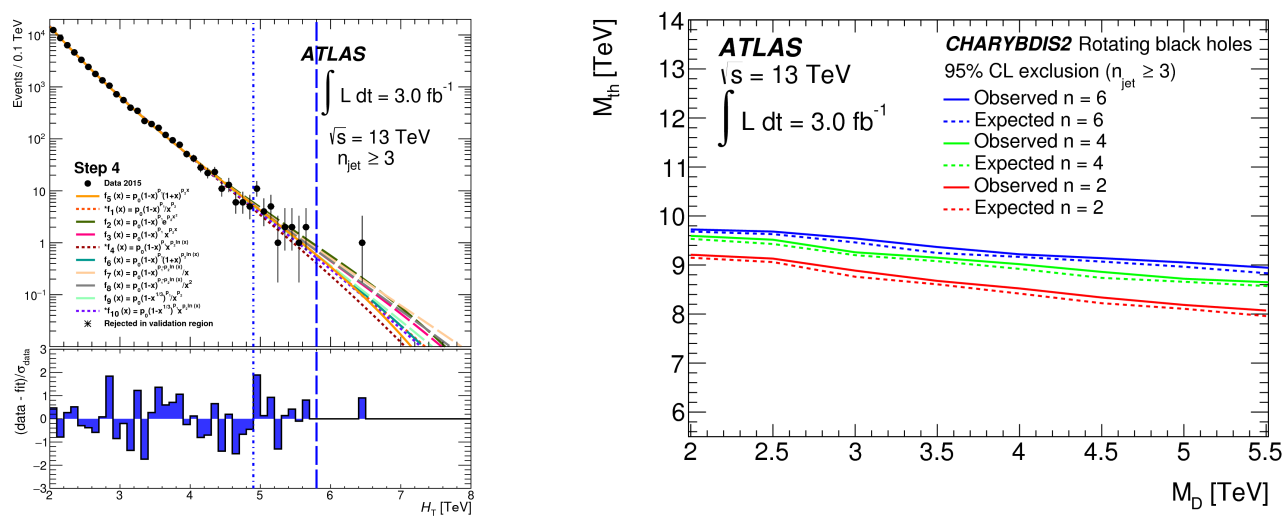

Figure 5: Scalar sum of the jet $p_{T}$ (left panel). The background model relies on a fit-based technique as explained in the text. The vertical lines show control, validation and signal regions. The right panel shows observed and expected 95\% CL exclusion limits on rotating black holes with different number of extra dimensions in the fundamental Planck scale - threshold mass grid [7]. 


\section{Mixed final states}

The two last analyses covered in this report are the state with at least one lepton plus two additional objects (which can be leptons or jets) [12] and the lepton-jet resonances [13].

The signature for the first analysis is at least one lepton with $p_{T}>100 \mathrm{GeV}$ and two additional objects (either leptons or jets) with $p_{T}>100 \mathrm{GeV}$. The search observable is $\Sigma p_{T}$ of all leptons and jets with $p_{T}>60 \mathrm{GeV}$. Two signal regions are used in the analysis, with requirements $\Sigma p_{T}>2$ and $3 \mathrm{TeV}$. The SM background is described using a MC simulation. The $t \bar{t}, W+$ jets and $Z+$ jets background normalisation are taken from a simultaneous fit of background dedicated control regions. The $\Sigma p_{T}$ distribution for the electron channel is shown in Figure 6 (left panel). No significant excess is observed. Limits for the rotating black holes with two, four and six extra dimensions are set as a function of $M_{D}$ and $M_{t h}$, and can be seen in Figure 6 (right panel).
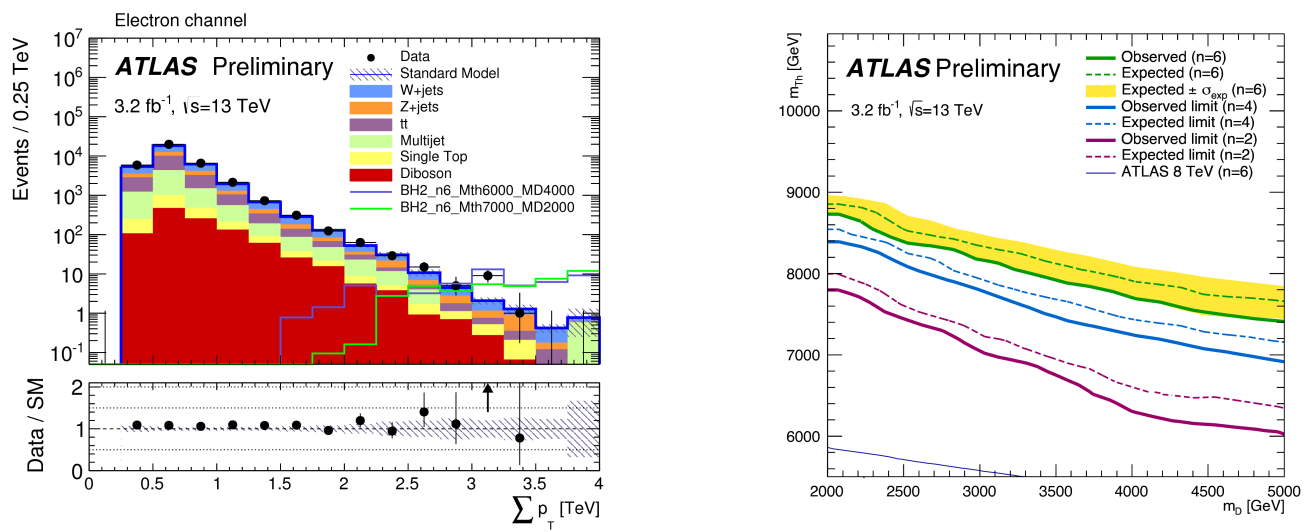

Figure 6: The $\Sigma p_{T}$ distribution for the electron channel. Two representative signal distributions for the rotating black holes with six extra dimensions are overlaid (left panel). The right panel shows exclusion contours in the fundamental Planck scale and threshold mass plane for the rotating black hole models with two, four and six extra dimensions [12].

The final analysis discussed in this report is the search for lepton-jet resonances, which is an inclusive search for new physics signatures of such resonances. The analysis signature consists of two electrons (muons) with $p_{T}>30(40) \mathrm{GeV}$ and two or more jets with $p_{T}>50 \mathrm{GeV}$. Two lepton-jet pairs are formed from these objects in a way that the invariant mass difference between the two pairs has to be the smallest. The lower invariant mass of these two pairs, $m_{L Q}^{\min }$, is the search variable for the analysis. The SM background is described by a MC simulation and $t \bar{t}$ and DY+jets background normalization are taken from a simultaneous fit in the background-dedicated CRs. The $m_{L Q}^{\min }$ distribution for the electron channel is shown in Figure 7 (left panel).

The tested BSM model for the analysis is the pair production of first- and second-generation scalar leptoquark (minimal Buchmüller-Rückl-Wyler model). Upper limits on the cross-section as a function of the leptoquark mass are shown in Figure 7 (right panel). The observed limit at 95\% $\mathrm{CL}$ is stronger by $50 \mathrm{GeV}$ comparing with the ATLAS Run 1 result, and corresponds to $1100 \mathrm{GeV}$ and $1050 \mathrm{GeV}$, for first- and second-generation leptoquarks, respectively. 

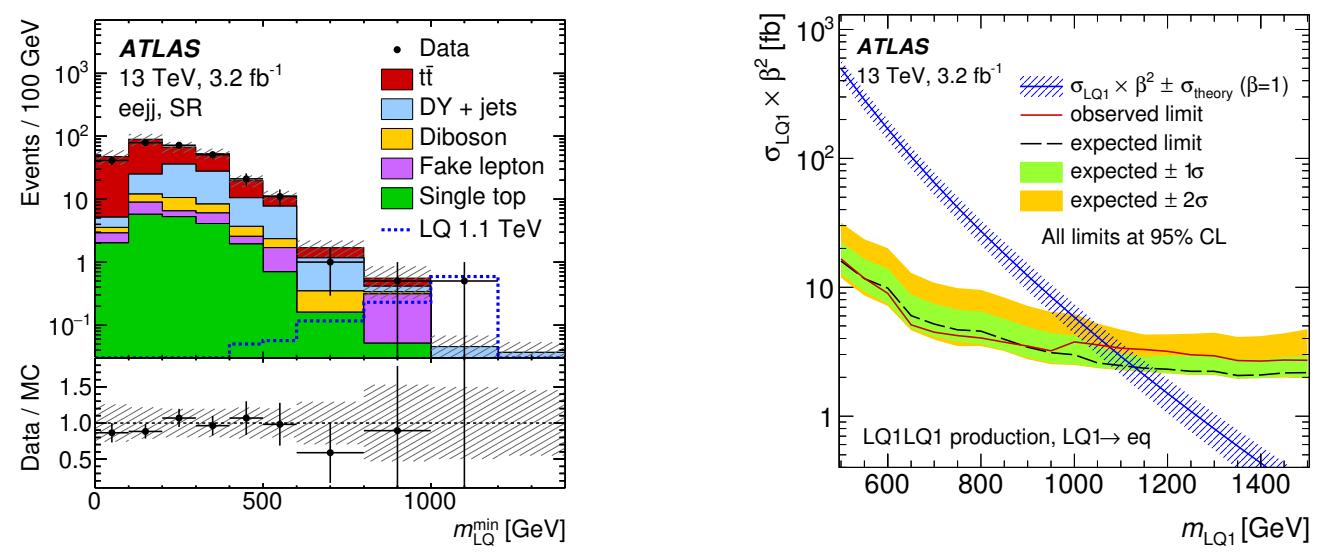

Figure 7: Distribution of the minimum reconstructed leptoquark candidate mass, $m_{L Q}^{\min }$, in the signal region of the first-generation leptoquark search. The signal expectation for a leptoquark of the mass of $1.1 \mathrm{TeV}$ is also shown (left panel). The cross-section limits on scalar leptoquark pair production times the square of the branching ratio as a function of mass are also shown (right panel) [13].

\section{Summary}

This report covers current ATLAS results on searches for new physics in high-mass fermionic final states and jets. The sensitivity of all such searches already now exceeds the Run 1 results, and new mass regions have been explored. No significant excesses are observed, but stronger exclusion limits are set for various models beyond the Standard Model. The expected ten times higher luminosity in 2016 will allow for a deeper exploration of the $13 \mathrm{TeV}$ regime.

\section{References}

[1] ATLAS collaboration, Search for new resonances in events with one lepton and missing transverse momentum in pp collisions at $\sqrt{s}=13 \mathrm{TeV}$ with the ATLAS detector, Tech. Rep. ATLAS-CONF-2015-063, CERN, Geneva, Dec, 2015.

[2] ATLAS collaboration, Search for new phenomena in the dilepton final state using proton-proton collisions at $\sqrt{s}=13 \mathrm{TeV}$ with the ATLAS detector, Tech. Rep. ATLAS-CONF-2015-070, CERN, Geneva, Dec, 2015.

[3] ATLAS collaboration, Search for beyond the Standard Model phenomena in e $\mu$ final states in $p p$ collisions at $\sqrt{s}=13 \mathrm{TeV}$ with the ATLAS detector, Tech. Rep. ATLAS-CONF-2015-072, CERN, Geneva, Dec, 2015.

[4] G. Altarelli, B. Mele and M. Ruiz-Altaba, Searching for New Heavy Vector Bosons in p $\bar{p}$ Colliders, Z. Phys. C45 (1989) 109.

[5] ATLAS collaboration, Search for new phenomena in dijet mass and angular distributions from pp collisions at $\sqrt{s}=13 \mathrm{TeV}$ with the ATLAS detector, Phys. Lett. B754 (2016) 302-322, [1512.01530].

[6] ATLAS collaboration, Search for resonances in the mass distribution of jet pairs with one or two jets identified as b-jets in proton-proton collisions at $\sqrt{s}=13 \mathrm{TeV}$ with the ATLAS detector, Phys. Lett. B759 (2016) 229-246, [1603.08791]. 
[7] ATLAS collaboration, Search for strong gravity in multijet final states produced in pp collisions at $\sqrt{s}=13$ TeV using the ATLAS detector at the LHC, JHEP 03 (2016) 026, [1512 . 02586].

[8] R. M. Harris and K. Kousouris, Searches for Dijet Resonances at Hadron Colliders, Int. J. Mod. Phys. A26 (2011) 5005-5055, [1110.5302].

[9] D. M. Gingrich, Quantum black holes with charge, colour, and spin at the LHC, J. Phys. G37 (2010) 105008, [0912.0826].

[10] U. Baur, M. Spira and P. M. Zerwas, Excited-quark and-lepton production at hadron colliders, Phys. Rev. D 42 (Aug, 1990) 815-824.

[11] C.-W. Chiang, T. Nomura and K. Yagyu, Phenomenology of $E_{6}$-Inspired Leptophobic Z' Boson at the LHC, JHEP 05 (2014) 106, [1402 . 5579].

[12] ATLAS collaboration, Search for TeV-scale gravity signatures in high-mass final states with leptons and jets with the ATLAS detector at $\sqrt{s}=13 \mathrm{TeV}$, Tech. Rep. ATLAS-CONF-2016-006, CERN, Geneva, Mar, 2016.

[13] ATLAS collaboration, Search for scalar leptoquarks in pp collisions at $\sqrt{s}=13$ TeV with the ATLAS experiment, 1605.06035. 